\title{
È POSSIBILE UNA FILOSOFIA DELLA MENTE IN ARISTOTELE? ${ }^{1}$ \\ Barbara Botter (PUC-RJ) ${ }^{2}$ \\ barbarabotter@gmail.com
}

Resumo: Nei tre libri del De Anima Aristotele presenta differenti argomenti di interesse filosofico e scientifico in relazione alla natura della vita, al movimento, ai sensi, alla percezione e immaginazione, al pensiero e alla relazione fra mente e corpo. De Anima sembra, pertanto, una accozzaglia di teorie filosofiche e biologiche che solo marginalmente potrebbero impressionare un ricercatore contemporaneo. Nondimeno, questa impressione è solo superficiale, in quanto l'unità dell'opera è fornita da una acuta analisi della natura dell'essere vivente e delle sue funzioni proprie. Lo studio di Aristotele si rivela, perciò, un contributo significativo nel moderno dibattito nella filosofia della mente.

Palavras-chave: anima, mente, corpo, pensiero.

\section{L'ORIGINE DEL PROBLEMA}

La filosofia della mente trae origine dall'attenzione prestata alla speciale natura della facoltà mentale dell'uomo, perciò dall'esame di una attività peculiare di alcuni tra gli esseri viventi. Scopo di tale indagine è di distinguere l'attività corporea dall'attività mentale e di caratterizzare e circoscrivere la peculiarità di questa attitudine rispetto ai processi di natura biologica e fisica in generale.

\footnotetext{
${ }^{1}$ Recebido: 15.12.2009/Aprovado: 26.02.2010/Publicado on-line: 13.03.2011.

${ }^{2}$ Barbara Botter é Professora-adjunta do Departamento de Filosofia da Pontifícia Universidade Católica do Rio de Janeiro, Rio de Janeiro, Brasil.
} 
La filosofia di Aristotele non è pensata per questo tipo di problema. Aristotele ci offre la migliore alternativa alle teorie dualiste e antidualiste della mente, quindi la migliore alternativa al problema che ha tormentato la filosofia in un persistente conflitto durato per più di tre secoli: il problema della relazione mente-corpo. Non ha senso chiedersi se Aristotele sia stato un dualista o meno; e ostinarsi a rimanere all'interno di questo contesto interpretativo significa perdere il vantaggio che il filosofo greco ha su di noi, ossia il fatto di collocarsi al di fuori della tradizione cartesiana e postcartesiana. Il punto non è tanto che per lo Stagirita il problema del rapporto fra l'anima e il corpo possa essere bypassato; semplicemente non ha senso che il problema venga posto in questi termini. In De Anima I 3 Aristotele chiude il capitolo indicando l'errore più grave commesso dai Physiologoi, ossia quello di aver sviluppato l'indagine sull'anima trascurando del tutto la natura del corpo. I filosofi presocratici congiungono anima e corpo ponendo l'una nell'altro, senza spiegare la causa di tale unione, ossia quali sono le condizioni necessarie affinché un determinato corpo sia un vivente. Aristotele spiega che "ì in virtù della relazione di comunanza (koinônia) che l'uno agisce e l'altro subisce e che l'uno muove e l'altro è mosso e nessuna di queste cose si verifica tra entità prese a caso. Costoro, invece, si sforzano soltanto di descrivere le proprietà dell'anima, ma non aggiungono nulla a proposito del corpo che le riceverà come se, in conformità con $i$ miti pitagorici, un'anima qualsiasi possa entrare in un corpo qualsiasi. Sembra infatti che ogni corpo abbia una forma e una configurazione propria. Essi si esprimono in modo simile a chi dicesse che 
l'arte del carpentiere entra nei flauti. Ė necessario, infatti, che la tecnica si serva degli strumenti e l'anima del corpo" (DA I 3, $\left.407^{b} 13-26\right)^{3}$. Affinché qualcosa agisca in altro, e affinché l'altro subisca l'azione del primo, è indispensabile che ci sia una certa relazione fra i due: è necessario che il secondo abbia in potenza ciò che il primo ha in atto, dal momento che non è una materia qualsiasi che è predisposta a ricevere una certa forma. L'anima necessita di un corpo organico affinché possa realizzare le sue proprie funzioni (DA II 1, 412 28).

Data questa premessa, si tratta di chiedersi:

1) se la questione della relazione mente-corpo, che tormenta i filosofi contemporanei, potrebbe essere stata posta ad Aristotele, quindi, in primo luogo, se i termini utilizzati nel dibattito contemporaneo siano sovrapponibili ai termini aristotelici;

2) quale sia il fondamento da cui Aristotele parte per chiarire la relazione dell'anima con il corpo e come egli la concepisce.

3) se Aristotele concepisce una attività propria della mente e indipendente dal corpo, così da inaugurare una "filosofia della mente”.

\section{IL SIGNIFICATO DEI TERMINI UTILIZZATI NEL DIBATTITO}

I filosofi al giorno d'oggi raramente parlano di "anima". Essi preferiscono il termine "mente". Nel suo saggio Body and

\footnotetext{
${ }^{3}$ Tutte le traduzioni dei passi aristotelici sono dell'autore.
} 
Soul in Aristotle, Richard Sorabji allerta di fronte alla possibilità di fraintendere il termine "mente" quando riferito ad Aristotele:

The word 'soul' may sound archaic to some moderne art, and people may be tempted to substitute 'mind'. But then they are likely to confine the functions of the soul to what we call mental acts, and this take them away from Aristotle's conceptions of the soul (SORABJI 1993, p. 164)

Al contrario, come Sorabji persuasivamente argomenta, "we ought to recognize Aristotle's conception of soul [as] coextensive with life, that is, with all life" (SORABJI 1993, p. 165).

Una delle fonti che stanno all'origine della svolta linguistica anima/mente è certamente Descartes. Descartes utilizza entrambe i termini per riferirsi al medesimo fenomeno. Se egli manifesta una certa preferenza per il termine "mente", ciò è dovuto al fatto che egli rifiuta una certa concezione dell'anima, ossia quella di origine aristotelicoscolastica, che desidera sostituire con il concetto da lui coniato. La nozione di "anima" che Descartes rifiuta non è, a parlare propriamente, il principio aristotelico, bensì un suo derivato storico. Però è vero che la nozione rifiutata da Descartes condivide con l'idea aristotelica esattamente la caratteristica che il filosofo francese rifiuta, ossia la pretesa che l'anima sia ciò in virtù di cui un corpo è vivente (ARISTOTELE, DA II 2, 414 14-15; II 2, 4144²8-30; II 4, $\left.415^{\mathrm{b}} 8-12\right)$. Descartes, al contrario, ritiene che i corpi, viventi e non viventi, siano parte del mondo fisico e che possano essere spiegati senza fare alcun riferimento ad elementi spirituali, tali quali l'anima.

A dire il vero, però, non è interamente esatto affermare che Descartes rifiuta la nozione aristotelica di anima, bensì 
sarebbe più precisa l'affermazione contraria, se non fosse un controsenso dal punto di vista storico. Infatti, il concetto che Descartes adotta discende da un modello che Aristotele critica. E la nozione di Descartes condivide con la dottrina ripudiata da Aristotele esattamente quella caratteristica che il Maestro del Liceo critica. La concezione che Aristotele nega è quella platonica, secondo la quale l'anima non è solo ciò in virtù di cui un corpo è vivente, ma è soggetto proprio di ciò che potremmo definire le funzioni mentali. Secondo questa concezione l'anima è propriamente il soggetto che pensa o si impaurisce, mentre il corpo prova questi sentimenti solo in modo derivato. La nozione di anima attaccata da Aristotele è l'antecedente storico della nozione cartesiana di "mente". Dal punto di vista aristotelico, questa nozione è errata per almeno due ragioni:

1) non riesce a rendere conto della vita di un organismo, poiché riposa sull'assunzione che le funzioni vitali possono essere spiegate ricorrendo alla materia e alle sue proprietà;

2) presuppone che un corpo naturale non sia in grado di svolgere funzioni quali desiderare, o provare sensazioni e per questa ragione richieda l'intervento di un'anima.

Quindi, i termini "mente" e "anima" non sono immediatamente sostituibili in quanto non ricoprono lo stesso significato.

Il secondo termine del dibattito è "corpo", nel senso di materia corporea. Per Aristotele ogni genere di essere vivente ha un tipo di materia, una materia prossima, ossia una 
materia propria a quell'ente dotato di vita ${ }^{4}$. Le potenzialità della materia prossima sono peculiari ad essa e non derivano dalle potenzialità degli elementi di cui la materia prossima è costituita. In altri termini, non c'è per Aristotele un concetto generico di materia e non c'è una materia unica per tutti gli esseri naturali. La soluzione aristotelica differisce da quella moderna incline, invece, a presupporre un concetto monolitico di materia, sulla scia della proposta cartesiana.

Descartes rifiuta l'idea che un corpo fisico possa contenere un principio interno di movimento e rigetta la pretesa che possano esistere corpi fisici definiti ognuno da una loro natura propria. Per il filosofo francese la natura di un corpo si riduce all'estensione di esso e questa qualità fisica non può costituire un principio interno di mutamento. Il corpo, di conseguenza, è inerte. La conclusione cartesiana deriva, quindi, dalla combinazione di due premesse:

1) l'idea aristotelica che, se la potenza attiva e la potenza passiva vengono escluse dal corpo fisico, ciò che rimane è l'estensione, ossia per Aristotele la lunghezza e la larghezza (Metaph., VII 3, 1029a11-19);

\footnotetext{
${ }^{4}$ Aristotele, (DA II 1, 412 $\left.{ }^{\mathrm{b}} 4-25\right)$; cfr. Metaph., (VII 11, 1036 22 sg., in particolare 1037 24-33). Le accezioni "materia prossima" e "materia remota" non sono aristoteliche, ma sono state adottate per risolvere alcune incongruenze dei testi aristotelici che sembrano a volte non distinguere una materia considerata in senso assoluto, e che viene esclusa dalla definizione dell'ente vivente, e il carattere correlativo del termine materia, secondo il quale la materia deve essere inclusa nella definizione. "Materia prossima" indicherebbe, in altri termini, le parti omeomere di un ente, mentre la "materia prossima" indicherebbe le parti organiche (cfr. IRWIN 1988, § 131). Questa proposta, tuttavia, ignora alcune difficoltà, come per esempio il fatto che in Metaph. (1035 $\left.5^{\mathrm{b}} 10-11\right)$ Aristotele, contrariando la pretesa di Irwin, esige che il dito, parte organica e non omeomera e, perciò, materia prossima, sia escluso dalla definizione. Ci pare più convincente la distinzione tra "materia che compone" e "materia funzionale" proposta da J. Whiting (1992, pp. 77-85) e adottata da S.M. Cohen (1992, pp. 69-70). Cfr. Botter (2009, pp. 113-126).
} 
2) l'idea non aristotelica che i corpi non hanno una loro natura propria e perciò nessuna potenza attiva o passiva.

Il corpo cartesiano è il corpo aristotelico privato di tutte le sue potenzialità, perciò niente altro che lunghezza e larghezza, quindi estensione.

Volendo approssimare Aristotele al dibattito contemporaneo sul rapporto mente-corpo, la soluzione aristotelica non rientra in nessuna delle alternative proposte dalla filosofia della mente, mantenendosi lontana da ogni genere di dualismo cartesiano o materialismo (Cfr. SEARLE, 1992, p. 204). Nel saggio The throughly Modern Aristotle: Was he really a functionalist?, Christopher Green nota che "the debate over what sort of theory Aristotle proposed has, at least over the last few decades, pretty well tracked the debate about what sort of a theory of mind is actually true" (GREEN 1997, p. 2). Green ha persuasivamente argomentato che neppure la moderna interpretazione della filosofia aristotelica come una sorta di tesi funzionalista è adeguata, dal momento che il filosofo greco non avrebbe accettato "the transportability thesis", tanto essenziale per il funzionalismo ${ }^{5}$. Nel Postscript a Body and Soul in Aristotle, Richard

\footnotetext{
${ }^{5}$ La soluzione funzionalista venne proposta da Hilary Putnam nel (1975) e alcuni anni più tardi da Martha Nussbaum nella sua edizione del De Motu Animalium del (1978). Putnam dichiarò una certa ispirazione aristotelica nella sua proposta di intendere gli "stati mentali" come "stati funzionali della materia". Martha Nussbaum riprese con maggiori dettagli questa interpretazione di stampo "funzionalista", ora non più ristretta al dominio della filosofia della mente, ma estesa all'intero dominio della natura. Secondo l'interpretazione funzionalista, la forma sarebbe definita da un complesso di funzioni articolate e potrebbe essere realizzata in diversi tipi di materia eterogenei fra loro. Questa posizione si propose come una alternativa alla lettura di Ackrill (1979), il quale, esaminando la definizione dell'anima esposta in (DA II 1), espose con invidiabile chiarezza il problema dell'indiscernibilità tra materia e forma negli esseri viventi, che Aristotele indica col nome di ousia. Secondo Ackrill, la distinzione fra materia e forma trova origine nella necessità di risolvere alcuni paradossi presenti nelle descrizioni del movimento. Nella sfera biologica, tuttavia,
} 
Sorabji argomenta che la lettura della dottrina dell'anima come un congiunto di capacità, da lui adottata, "has been wrongly taken to endorse a functionalist reading of Aristotle's philosophy of mind" (SORABJI 1993, p. 186), che lo studioso esplicitamente rifiuta.

Esaminando la possibilità di una interpretazione dualista della teoria aristotelica è facile rimanere vittima di un fraintentendimento. Parlare di "anima" e "corpo" dà l'impressione che si possa disquisire intorno all'anima come se fosse una sostanza capace di esistere indipendente dal corpo, come nel caso di Platone (Fedone 64') e Descartes (1988, p. 25). Ma la concezione aristotelica dell'anima come forma di un organismo dotato di vita impedisce questa lettura. L'organismo vivente nella sua completezza è la sostanza prima per Aristotele, e questo organismo non è l'unione della sostanza prima dell'anima e della sostanza corporea ${ }^{6}$.

D'altro lato, la filosofia aristotelica preclude l'accesso al materialismo. Nel capitolo 1 del libro I del De Anima lo Stagirita sembra accettare una concezione materialista degli stati emozionali esprimendosi a favore di una concezione della rabbia come di un ribollire del sangue nella zona intorno al cuore (DA I 1, 40331-32). Tuttavia ogni

\footnotetext{
il "principio di omonimia", esigendo la presenza di una materia capace di esercitare le funzioni richieste dall'organismo, toglierebbe alla suddetta distinzione qualunque potere esplicativo, dal momento che materia e forma si configurano come due aspetti dell'analisi di uno stesso oggetto e si riferiscono ad un unico ente. Recentemente Burnyeat (1992) riprese l'argomento di Ackrill e tentò di mostrare che l'hylemorfismo aristotelico non permette una "specificazione contingente della materia" e non ammette la "plasticità funzionale della forma". Per lo studioso, l'hilemorfismo aristotelico è rigorosamente incompatibile con i nuovi modelli di spiegazione scientifica emersi a partire dal secolo XVII, dal momento che non riconosce una materia che possa essere compresa in se stessa, indipendentemente dalla forma dell'animale.

${ }^{6}$ Con sostanza "prima" alludiamo alla dottrina del capitolo 5 delle Categorie $\left(2^{\mathrm{a}} 11-4^{\mathrm{b}} 19\right)$ e, più precisamente, al terzo senso di sostanza come sinolo di materia e forma (DA II 1, 412 6-9).
} 
interpretazione materialista è scoraggiata dalle linee che seguono il passo in questione. Aristotele spiega chiaramente che qualsiasi soluzione puramente fisica del problema risulterebbe incompleta, non diversamente da una spiegazione puramente psicologica (DA I 1, 403 30-31). Un tentativo adeguato consiste nel combinare entrambe le componenti, la componente fisica e quella psicologica (DA I 1, $403^{\mathrm{b}} 1-2$; b7-8). La richiesta presenza di entrambe le condizioni preclude la strada non solo ad una teoria dell'identità, ma anche a qualunque tipo di riduzionismo. Una teoria dell'identità è rifiutata per il fatto di credere, per usare l'esempio di Aristotele, che pietre, mattoni e legno siano identici a "un riparo contro i danni provocati dal vento, dalla pioggia e dal calore". Ciò non vale per il fatto che i materiali costituenti una casa precedono alla costruzione e sopravvivono alla distruzione della casa stessa. Una teoria riduzionista, a sua volta, è vittima dell'idea che "un riparo contro le intemperie" non sia altro che "pietre e mattoni"? Ma nella dottrina aristotelica non è possibile ridurre le cause formali alle cause materiali.

Infine, ogni forma di dualismo parte dal presupposto dell'esistenza della mente come un dato di fatto e si pone il problema di fornire un ragguaglio accettabile intorno all'esistenza dei corpi. Questa è la ragione per cui Descartes nella seconda delle sue Meditationes argomenta favorevolmente intorno all'esistenza delle menti, ma prende in considerazione la condizione del corpo solo nella sesta meditazione (1988, pp. 24-25, 71, 80). Il materialismo, a sua volta, parte dal presupposto inverso di considerare l'esistenza dei corpo come un dato di fatto e si chiede come

\footnotetext{
${ }^{7}$ L'esempio, fornito dallo stesso Aristotele, si trova in (DA I 2, 403a29-b16).
} 
sia possibile un accesso alla mente. Il problema viene formulato da Jaegwon Kim in Philosophy of Mind: "the basic mind-body problem is that of accounting for the place of mind in a world that is essentially physical" (KIM, 1998, p. 9).

A partire dalla separazione dell'organismo vivente in due sostanze eterogenee, l'una evidente, l'altra misteriosa, qualsiasi tentativo di risolvere il problema mente-corpo si rivela un compito erculeo. Noi crediamo, pertanto, che l'intento di concentrare l'indagine sull'organismo vivente nella sua interezza possa costituire un approccio proficuo alla questione della natura e della relazione fra anima e corpo. A partire dalla prospettiva fortemente biologica di Aristotele, ogni organismo vivente è una unità, al punto che qualsiasi divisione tra anima e corpo o tra le parti dell'anima sembra pura astrazione (Cfr. HAMMOND, 1902, p. XXI ). Del resto, nel libro VIII al capitolo 6 della Metafisica, Aristotele ci mette in guardia sulla difficoltà che inevitabilmente si crea quando si pretenda indagare l'unione di sostanze distinte. Aristotele spiega che giustificare l'unità si rivela impossibile se si parte dal presupposto che essa risulti dalla somma delle eterogenee caratteristiche di un ente (Metaph. VIII 6, 1045ª 7-20). Per la stessa ragione, il problema dell'unità mente-corpo resterà un dilemma irrisolto se per prima cosa si divide in due l'essenziale unità dell'ente dotato di vita.

\section{IL FINE DELL'INDAGINE ARISTOTELICA}

Passiamo ora al secondo punto, qual è precisamente l'oggetto dell'indagine aristotelica.

Nella sua ricerca intorno alla natura dell'essere umano, 
Aristotele non sembra attribuire una enfasi particolare al problema del "mentale". La sua preoccupazione sembra essere piuttosto quella di soddisfare la richiesta di Wittgenstein, "di avere una storia naturale dell'uomo" (1956).

L'idea che sta al centro della ricerca aristotelica è quella di caratterizzare il principio che rende gli esseri viventi differenti dagli artefatti e di mostrare le diverse nature dei differenti enti naturali. La soluzione del problema è racchiusa nel termine chiave "natura" di un essere vivente (physis) $)^{8}$. La "natura" è ciò che permette di intendere che cosa è un ente e in cosa un ente differisce da altri enti. Il termine physis non è facilmente traducibile e non è immediatamente sostituibile con il termine "fisico", almeno così come esso è utilizzato nel dibatto contemporaneo, benché Aristotele, da parte sua, non avrebbe avuto nessuna difficoltà a glissare fra i due termini, tanto che, all'inizio del De Anima, egli afferma che lo studio della natura umana è compito del physikos (DA I 1, 40325-30). Physikos è colui che studia ta physika, ossia i fenomeni fisici. Tuttavia, nella concezione moderna il fisico è sottostimato al punto tale che generalmente si tende a pensare ad esso come ai soli materiali costituenti e alle proprietà di essi. Un tale impoverimento del lato fisico crea una considerevole pressione ad introdurre qualcosa di non fisico per rendere ragione dei fenomeni mentali.

Se "natura di un ente" non è ciò che "fisico" nella lingua corrente denota, di cosa si tratta allora?

\footnotetext{
${ }^{8}$ L'importanza di questo termine è testimoniata anche dal fatto che esso ricorre ben 265 volte nel De Partibus Animalium. Solo altri due termini sono usati dal filosofo così frequentemente da superare le 200 ricorrenze, ossia il termine zôon e un termine che gioca un ruolo peculiare nel De Partibus Animalium, ossia morion.
} 
La natura di un ente è ciò che permette di capire perché un animale razionale differisce da un animale senziente e un animale senziente da un essere vivente e un essere vivente da un corpo naturale e un corpo naturale da un artefatto (Cfr. ARISTOTELE, DA II 2, 413 23-26). La natura di un ente è, quindi, anche un principio esplicativo, ma la sua funzione non si esaurisce a ciò. L'interesse di Aristotele consiste nel discernere la causa per cui gli enti del mondo sublunare e particolarmente gli esseri viventi sono ciò che sono. Questa causa è detta "natura" (physis) nella Fisica e ousia ${ }^{9}$ nella Metafisica ${ }^{10}$. Per Aristotele la "natura" di un corpo animato è la sua ousia, ossia, la causa del suo essere. Per gli enti sensibili, causa dell'essere è la forma, ossia l'anima, ma si tratta di una forma capace di sussumere la materia come elemento costituente la sua propria attualità. È la presenza dell'anima che conferisce ad un certo corpo la sostanza di animale (DA II 1, 412 16 -22, b11-26; PA I 1, 641 18-21): "è necessario che l'anima sia sostanza come forma di un corpo naturale che potenzialmente possiede vita. E la sostanza è atto. Perciò è di un corpo di questo tipo che l'anima è atto"

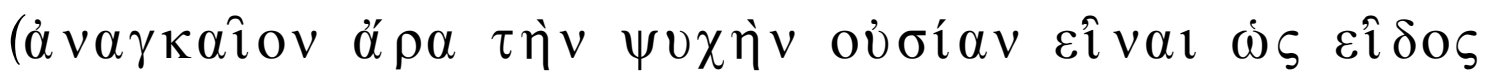

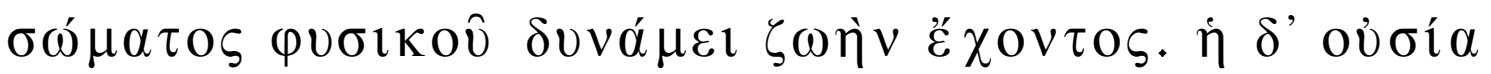

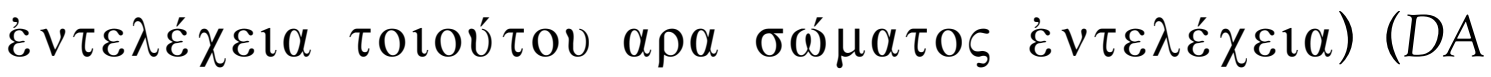
II 1, 412 $19-22)$ E dire che l'anima è l'essenza di un corpo

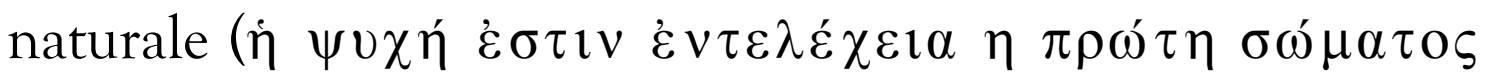

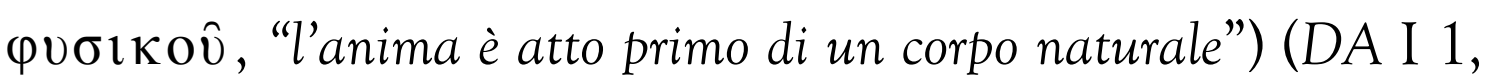

\footnotetext{
${ }^{9}$ Nella costruzione "ousia di qualcosa”.

${ }^{10} \mathrm{Al}$ di là di questa divergenza lessicale, esiste una piena convergenza fra le due opere, prova ne è che nella conclusione di Metafisica VII, ousia di qualcosa è designata come "natura" (Metaph. VII 17, 1941 30 ); in Fisica II 1 il filosofo utilizza i termini l'uno accanto all'altro ("alcuni ritengono che la natura e l'essenza degli enti naturali", Phys. II 1, 193²9-10); e in De Partibus Animalium (I 1, $641^{a} 25$. 27) si dice che la natura è precisamente l'ousia.
} 
412 27), significa affermare che il corpo, inteso come un

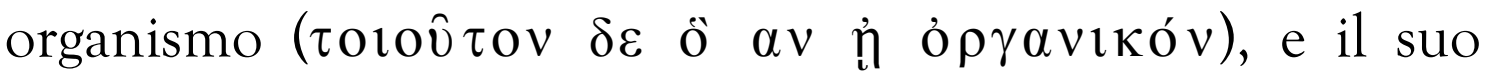
comportamento devono essere concepiti in un certo modo se si vuole autenticamente comprenderli. La presenza dell'anima è la presenza dell'atto primo di tutte le funzioni proprie dell'animale, ossia è la forma dell'animale intesa come un insieme articolato di funzioni e strutture e non come una misteriosa entità che si radica nella "macchina" corporea. L'anima è la capacità di svolgere in modo adeguato tutte le funzioni e attività proprie di un animale attraverso il corpo naturale. La natura di un ente permette di capire una condotta peculiare, ma soprattutto di rendere conto delle ordinarie funzioni di un ente $(v \hat{v} v \delta$ ' $\dot{\varepsilon} \pi \grave{i}$

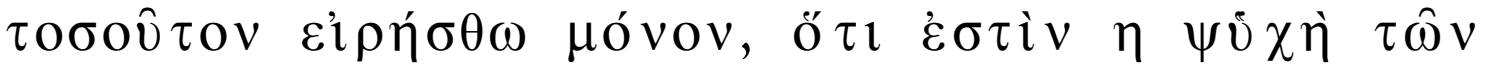

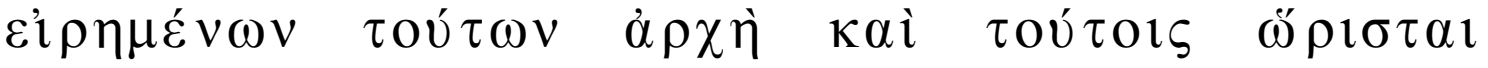
$\theta \rho \varepsilon \pi \tau \imath \kappa \omega, \alpha \imath \sigma \theta \eta \tau \imath \kappa \hat{\varphi}, \delta \imath \alpha \nu 0 \eta \tau \imath \kappa \hat{\varphi}, \kappa \imath v \eta ́ \sigma \varepsilon \imath$. "per ora $\dot{e}$ sufficiente dire solo questo, che l'anima è principio delle capacità menzionate, nutritiva, percettiva, razionale e di movimento, ed è definita da esse") (DA II 2, 413 1 11-13).

Aristotele ritiene che non ci sia alcuna ragione per trattare le cosiddette funzioni mentali, come desiderare, pensare, credere, e così via, in un modo diverso dalle altre funzioni della vita ordinaria. Parlando delle affezioni dell'anima (pathe), egli afferma che esse coinvolgono il corpo e che non sono peculiari all'anima, in quanto le affezioni sono formulae nella materia $(\delta \hat{\eta} \lambda \mathrm{\gamma} v$ ö $\tau \imath \tau \dot{\alpha} \pi \alpha \dot{\theta} \theta \eta$

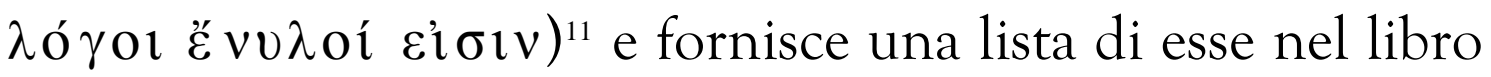
II del De Anima: nous, dianoetikon = pensare; aisthesis =

\footnotetext{
${ }^{11}$ Aristotele, (DA I 2, 403²5). Cfr. l'espressione $\tau \delta \delta \varepsilon \dot{\varepsilon} v \tau \hat{\varepsilon} \delta \varepsilon$, "la forma presente in una materia" di (PA I 1, 640 26 ) che designa propriamente la struttura hylemorfica di un composto; e ancora Metaph. (VII 5, 1030 18 ; VII 11, 1036 23); (DA III 4, 429 14 ).
} 
provare sensazioni; kata topon kinesis = muoversi in un luogo; stasis = essere fermo; kata trophen kinesis = muoversi, $\mathrm{ma}$ in rapporto alla nutrizione; auxesis = crescere; orexis, thumos, boulesis = desiderare; genesis = riprodursi (DA II 2, 414 $18-21$; cfr. I 1, 403 7-10; I 5, 411 27-b3). In De Sensu il filosofo ribadisce che "le funzioni più importanti, sia comuni, sia proprie degli animali, sono chiaramente comuni all'anima e al corpo (DA III 10, 433 $\left.{ }^{\mathrm{b}} 19-20\right)$, come la sensazione, la memoria, la passione, il desiderio e, in generale, l'appetito, e inoltre il piacere e il dolore: queste appartengono più o meno a tutti gli animali". Facciamo inoltre notare che il breve trattato, cui è stato attribuito il titolo di Parva Naturalia, assieme al De Motu si riferiscono a ciò che Aristotele designa con il titolo Funzioni condivise dall'anima e dal corpo. L'esordio al primo di questi trattati, Sulla Percezione, sembra suggerire che l'intero ambito delle funzioni che saranno discusse in questi trattati siano comuni all'anima e al corpo. Aristotele rifiuta di dividere questa lista. Egli pensa piuttosto che in natura esista un ente, il quale è in grado di compiere tutte le suddette attività attraverso il suo corpo organico in virtù della sua "natura".

Nel capitolo 1 del libro II della Fisica, Aristotele definisce precisamente il termine "natura" come principio immanente di movimento e di riposo e afferma che "ha natura tutto ciò che possiede tale principio" (ARISTOTELE, Phys. II 1, 192 ${ }^{\mathrm{b}} 32$ ). Dopo aver proposto questa definizione (Phys. II 1, 192 $2^{\mathrm{b}}$-19399), il filosofo indica due nozioni che soddisfano tale descrizione (Phys. II 1, 193 ${ }^{\mathrm{a}} 9-\mathrm{b} 6$ ): da una parte la materia, dotata di movimenti necessari legati alla natura propria degli elementi di cui è composta; dall'altra la forma, nella misura in cui a titolo di causa finale governa e amministra la concatenazione dei movimenti necessari causati 
dalla materia elementare (Phys. II 1, 193²8-31, II 2, $194^{\mathrm{a}} 12$ 13).

Dopo aver attribuito tanto alla forma quanto alla materia il titolo di "natura", Aristotele formula il problema di sapere a quale di queste due "nature" lo studioso di scienza naturale deve prestare attenzione. Opzioni unilaterali, esclusivamente per la forma o esclusivamente per la materia, sono presto scartate e lo Stagirita formula ciò che si conosce sotto il nome di hylemorfismo teleologico. Affinché si costituisca un organismo dotato di vita non è sufficiente assumere gli attributi essenziali degli elementi materiali come presupposto, dal momento che i movimenti della materia non riuscirebbero a produrre le proprietà richieste dalle funzioni dell'organismo, in altri termini, essi non sono sufficienti a riprodurre la vita. Ė necessario l'intervento di un principio anteriore che, in qualità di causa finale, sia in grado di sviare dal loro corso spontaneo le serie dei movimenti degli elementi materiali. Questo principio è propriamente la forma dell'animale, che nel caso dell'anima, converge con le cause finale ed efficiente (DA II 4, 415 8-14; PA I 1, 641ª 27; Phys. II 7, 198 24-27). In quanto causa formale (eidos, forma), l'anima è essenza (ousia) dell'animale e natura (physis). Questa natura è precisamente (i) dal punto di vista del venire all'essere, "ciò in vista di cui" si originano i processi generativi e ciò in cui risultano tali processi (ARISTOTELE, Metaph. VII 7, 1032²1-25); e (ii) dal punto di vista dell'essenza, il fine in cui l'essere vivente attinge il suo pieno sviluppo. A sua volta, questo fine deve essere inteso anche come causa efficiente, e ciò in sue sensi: (i) dal punto di vista del venire all'essere, è la forma del genitore che determina la sequenza dei movimenti che risulteranno in un individuo della stessa specie: e (ii) dal 
punto di vista dell'essere, la forma si presenta come il congiunto delle capacità grazie alle quali l'essere vivente svolge tutte le attività che lo caratterizzano essenzialmente, dalla nutrizione al pensiero ${ }^{12}$.

L'anima interviene nelle interazioni elementari degli elementi in modo tale che essi acquisiscono proprietà accidentali e si armonizzano in combinazioni che trasgrediscono le leggi originarie della materia ${ }^{13}$. Una volta determinati secondo queste proprietà accidentali, gli elementi assumono la configurazione di ciò che Aristotele chiama "materia propria" 14 dell'essere vivente. Tra la materia propria e la forma dell'animale (anima) esiste una relazione di implicazione reciproca (Metaph. VIII 6, 1045 33), al punto da essere fra loro indiscernibili: $\varepsilon^{\circ} \sigma \tau \imath \delta^{\prime}$

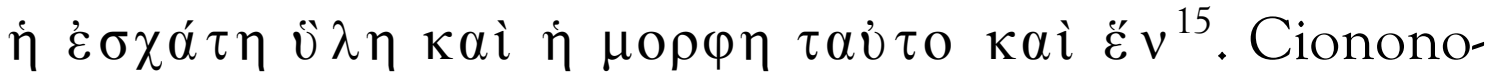
stante, la materia propria è costituita di elementi che preservano, sebbene solo potenzialmente (ARISTOTELE, PA II 3, 649 10 -20), le loro disposizioni originarie e voltano allo stato originario con la morte dell'individuo ${ }^{16}$. L'anima

\footnotetext{
${ }^{12}$ Aristotele, (DA II 4, 415 $21-27$ ). Intorno all'importanza centrale che lo Stagirita attribuisce all'anima come punto di convergenza di tre tipi di causa vd. Balme (1992, p. 90); Nussbaum (1978, pp. 78-80 e 1980, p. 363); Charles (1991, pp. 126-127); Sauvé (1992, p. 816).

${ }^{13} \mathrm{Vd}$. l'esempio del sangue in (PA II 3, 649 20-34); vd. anche (DC II 6, 288 $15-18$ ). Angioni (1999), commento al passo in questione.

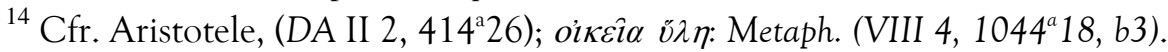

${ }^{15}$ Aristotele, (Metaph. VIII 6, 1045 17-19). Materia propria e forma sono distinti come "aspetti" di una stessa cosa, l'uno in potenza, l'altro in atto.

${ }^{16}$ Cfr. Aristotele, (DC II 6, 288 $15-18$ ): "e tutte le debolezze (o incapacità, adynamiai) negli animali sono contro natura (para physin), come la vecchiaia e l'indebolimento. Infatti, l'intera costituzione degli animali si forma a partire di elementi che differiscono dai loro propri luoghi naturali, poiché nessuna delle parti (le porzioni di elementi che costituiscono l'animale) occupa il luogo che gli è proprio". Ė chiaro che tra la materia che mantiene i propri attributi essenziali indipendentemente dalla forma dell'essere vivente e la forma stessa non esiste nessuna implicazione reciproca, e, pertanto, non c'è quella indiscernibilità che tanto infastidì Ackrill (1979) e che generò nella letteratura secondario il famoso "problema di Ackrill". I tentativi di risolvere il problema di Ackrill tentano di provare che la relazione fra materia e forma è, per così dire, accidentale, nella misura in cui la forma è un predicato che sopravviene alla materia. Vd. Burnyeat (1992); Kuhn (1978, pp. 155-156); Furth (1988,
} 
amministra i movimenti della materia secondo un presupposto che è loro estrinseco, quindi svia alcuni di questi moti dal loro corso originario conferendo all'ente gli attributi dell'essere vivente.

La teleologia, ossia l'impiego di cause finali come principio che dirige ed ordina adeguatamente la materia, lungi dall'introdurre fini estrinseci che dovrebbero essere acquisiti dagli enti naturali, si risolve in una analisi in cui il fine intrinseco ad ogni ente naturale, empiricamente constatato in un individuo adulto, è assunto come ragion sufficiente per discernere le proprietà e le condizioni materiali che devono essere soddisfatte da ogni ente naturale in atto. La teleologia offre una prospettiva in cui:

1) l'essenza di ogni ente è delimitata dalla sua funzione e attività propria, come lascia ben chiaro il principio di omonimia $^{17}$. Questo principio esprime precisamente la necessità di assumere la funzione propria dell'ente come principio del suo essere. Aristotele, dichiara che l'essenza di un item, sia esso un ente naturale o un artefatto, è determinata dalla funzione, ossia dalla capacità di eseguire l'attività che gli è propria attraverso il corpo o grazie alla materia di cui è composto. Perciò, secondo il principio di omonimia, sarebbe impensabile una separazione fra materia e forma in un corpo naturale ${ }^{18}$.

pp. 250-251); Loux (1991, pp. 54-64); Lewis (1991, pp. 146; 152-153); Brunschwig (1979).

${ }^{17}$ Aristotele, (PA I 1, 640 35-641 3 ; Meteorologica IV 12, 389 31 -390 2 ; DA II 1, 412 ${ }^{\mathrm{b}} 13-15$; 21; GA I 19, 726 22-24; II 1, 734 24-27; Metaph. VII 10, 1035 24-25; Alessandro d'Afrodisia, In Metaph. Ad $\left.1040^{\mathrm{b}} 5,769^{\mathrm{b}}\right)$.

${ }^{18}$ Questa unità intrinseca fra materia e forma genera, tuttavia, numerose difficoltà. Fu essa che generò il famoso "problema di Ackrill", formulato da Ackrill in Aristotle's Definition of Psuche e intorno al quale si organizza il dibattito attuale in relazione all'hylemorfismo e alla filosofia della mente di Aristotele. Dato il principio di omonimia, secondo il quale non è possibile una separa- 
2) la correlazione fra materia e forma è risolta in modo tale da evitare tanto l'indiscernibilità, così come una inconciliabile eterogeneità.

Questo secondo punto può essere più chiaramente inteso se si osserva che la teleologia non è incompatibile con il ricorso alla materia, ma la sussume. Ora, la materia dei corpi sublunari si risolve, in ultima istanza, nei quattro elementi (ARISTOTELE, DC III 1, 298 3-4; GC II 8, 334 30 -31), dotati di proprietà essenziali, da cui deriva un tipo di movimento che Aristotele caratterizza come un tipo di "necessità assoluta". La necessità assoluta delle cause materiali ed efficienti consiste nella necessità per cui, date le proprietà essenziali degli elementi, sempre necessariamente seguiranno certi effetti nelle stesse condizioni ${ }^{19}$. Ciò che Aristotele non ammette è che una unione spontanea $\mathrm{e}$ casuale di queste proprietà sia responsabile per ciò che maggiormente lo affascina, ossia la riproduzione continua delle specie (Vd. DA II 4, 415²5-b7).

Una volta ammesso che "entrambe le cause [la materia e la forma/fine] devono essere prese in considerazione dallo studio della natura" ${ }^{20}$, Aristotele si chiede a quale di esse si debba attribuire una sorta di primato e ribadisce che in modo particolare l'attenzione deve ricadere sulla causa finale, che è la forma (Phys. II 1, 198a24-27; PA , I 1, 640²2-29;

\footnotetext{
zione fra materia e forma nell'ente naturale, ci si potrebbe interrogare sulla possibilità di discernere i concetti di materia e forma, nati dall'analisi degli artefatti, nel dominio delle scienza naturali, in modo da oscurare i parametri attraverso i quali si di dice che l'hylemorfismo tende ad essere un dualismo attenuato, o un certo tipo di riduzionismo materialista, o ancora una sorta di compatibilismo o funzionalismo. Vd. Angioni (1999). Per un dibattito sulla questione, vd Nussbaum e Rorty (1992). ${ }^{19}$ Aristotele, (Phys. II 8, 198 12-14; II 9, 200²-5, 8-9, 14; GA II 4, 739 26-30; II 6, 743 a 36-b1; PA I 1, 640 8-11).

${ }^{20}$ Aristotele, (Phys. II 9, 200a32-34); la soluzione al problema della compatibilità fra cause materiali e finali é già suggerita in Fisica (II 2 194²1-29) e rinforzata in Fisica (II 7 198²2-24, b4-9).
} 
64125-27), e che merita il titolo di "natura" in modo preponderante ${ }^{21}$, per il fatto di essere causa motrice in modo prevalente. Da una parte, la forma deve ricevere maggiore attenzione per il fatto che essa è causa della materia e non viceversa $^{22}$; dall'altra, la forma/fine si traduce nella funzione (ergon) dell'ente e questa funzione indica l'atto proprio (energeia o entelecheia) in cui tale ente attinge la propria completezza. La forma è la causa per cui si spiega qualcosa che, apparentemente, non sarebbe suscettibile di spiegazione: la ragione per cui un ente è esso stesso, perché l'essere umano, per esempio, è essere umano.

Non si tratta di una tautologia, in quanto il senso dell'espressione è intendere la ragione per cui un essere umano è un animale di tal tipo, in cui "animale di tal tipo" definisce ciò che l'essere umano essenzialmente è. E la ragione per cui un essere umano è un "animale di tal tipo" è la forma. In quanto funzione, o complesso di funzioni, la forma spiega perché l'uomo possiede certi attributi essenziali ed è costituito di una materia con tali caratteristiche (Cfr. Metaph. 1041 b-6). È la forma intesa come funzione (ergon) ed atto (energeia) che è "causa della materia" in quanto essa spiega perché i movimenti necessari della materia devono

\footnotetext{
${ }^{21}$ Aristotele, (Phys. II 1, 193b-7: PA I 1, 64130-31). Il problema della predominanza delle cause formali/finali sulle cause materiali/efficienti deve essere inteso come un problema concernente l'alternativa fra "teleologia" e "necessitarismo". Per eccellenti e raffinate esposizioni di questa tematica vd. Nussbaum (1978 pp. 62-67), Waterloo (1982, pp. 62-80), Cooper (1987, pp. 262-264), Charlton (1985, pp. 136-145), Matthen (1989, p. 174), Charles (1988, pp. 5-8, 13, 17; 1991, pp. 102-104), Lewis (1988, pp. 54-58). Per una comprensione diversa nei dettagli, ma simile nel suo insieme vd. Gotthelf (1987, p. 212), Sauvé (1992, pp. 803, 822-824).

${ }^{22}$ Aition in questo contesto ha un significato forte: si tratta del fattore esplicativo preponderante. La stessa espressione aition tes hyles è attribuita alla forma in Metaph. (1041 $7-8$ e 1041 $\left.{ }^{\mathrm{b}} 30\right)$ : il filosofo afferma che la forma è natura, accanto alla natura concepita come elemento, stoicheion (1041³1). Vd. Angioni (1999).
} 
concatenarsi in un certo modo ${ }^{23}$. Il presupposto di Aristotele è che l'ente naturale è il risultato di una complessa combinazione di diverse catene causali. Fermo restando che una catena causale è una sequenza di interazioni di cause materiali ed efficienti, ciò che spiega la complessa e adeguata combinazione di esse è la causa formale/finale.

Nel libro I del De Partibus Animalium il problema della correlazione tra materia e forma, in altri termini la relazione fra anima e corpo, è esposto e risolto in modo meticoloso. Da un lato, si tratta di sapere se i movimenti necessari della materia siano sufficienti a spiegare gli organismi viventi in tutta la loro complessità ${ }^{24}$, come, secondo Aristotele, avrebbero preteso Empedocle e Democrito; dall'altro, Aristotele risolve tali questioni presentando il paradigma hylemorfico teleologico, che presuppone la relazione tra la forma, intesa come congiunto articolato di funzioni e strutture, e la materia, ossia i quattro elementi e i loro composti inanimati. Questo paradigma sottopone i movimenti necessari dei costituenti materiali alla direzione delle cause formali e finali. In altri termini, lo Stagirita si pone esattamente il problema di sapere se nelle spiegazioni scientifiche nell'ambito della natura il ricorso alla necessità assoluta, attribuita ai movimenti della materia, sia sufficiente o se sarebbe preferibile ricorrere ad un altro tipo di necessità che coinvolga la funzione e il fine dell'individuo, ciò che egli chiama "necessità

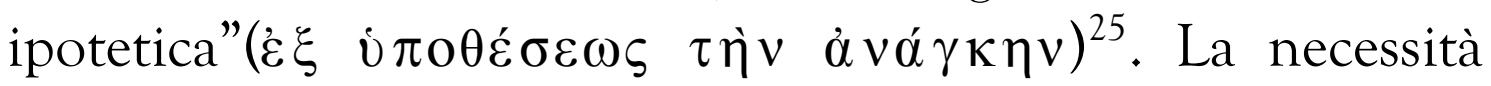

\footnotetext{
${ }^{23}$ Cfr. Aristotele, (Phys. II 2, 194ª 12-27; II 9, 200 30-35; 200b-8; Metaph. VI 1, 1025 $28-1026^{\mathrm{a}} 6$;

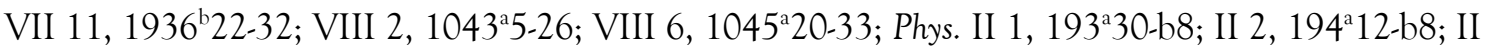
9, 200b33-35; PA I 1, 642 $9-13$.

${ }^{24}$ Cfr. Aristotele, (Phys. II 8 e 9), in particolare, (193ª-b8 e 194a 12-b8).

${ }^{25}$ Aristotele, (De somno 455 26; cfr. PA I 1, 639 21-640 10 ; 642 1-13; 642a31-b4; GC II 11, 337 9 -

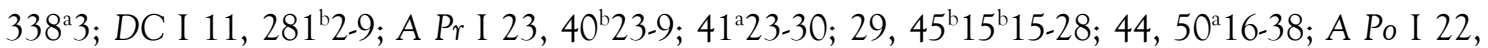


ipotetica, pur privilegiando la forma non disconosce le cause materiali, al contrario, le sussume come condizioni necessarie subordinate alla forma (sunaition) (ARISTOTELE, DA II 4, 416 14 ). I testi rilevanti a questo proposito si trovano nel De Partibus Animalium e nel De generazione Animalium. Tuttavia risultano decisivi i capitoli 8 e 9 del libro II della Fisica ${ }^{26}$. In questi testi Aristotele non nega l'importanza della materia nella costituzione degli esseri viventi ${ }^{27}$ : gli elementi materiali interagiscono fra loro secondo la necessità assoluta e questo comportamento è responsabile per certi movimenti e proprietà negli enti naturali, in modo tale che anche la materia vale come "natura", ossia come principio di movimento (Phys. II 1, 193228-31; II 2, 194 12-13). Nella misura in cui il corpo animale è composto di fuoco, sarà possibile attribuirgli certi attributi giustificabili solo attraverso le proprietà del fuoco (Vd. Aristotele, Phys. II 1, 193a28-30).

Ciononostante, la questione rilevante è sapere se la materia è responsabile per tutte le caratteristiche dell'ente naturale, o se esiste un altro principio, al di là della materia. Come risposta Aristotele introduce la "necessità ipotetica", che è la caratteristica di base della teleologia che egli attribuisce alla natura. Il filosofo ammette che le cause materiali possono generare un essere vivente solo presupponendo la forma, ossia la forma previa del genitore, "dal momento che è un essere umano che genera un essere umano". La forma agisce come un principio anteriore che amministra e governa le

\footnotetext{
$83^{\mathrm{b}} 38-84^{\mathrm{a}} 1$; II 6, 92 $\left.20-24\right)$.

${ }^{26}$ In particolare II 9, 200ª 5-10 (esposizione dell'argomento decisivo); 200ª 10-15 (esempi); 200³035 (ripresa dell'argomento); 200³5-b4 (nuovi esempi); 200 4-8 (corollari).

${ }^{27}$ Su questo punto vd. Cooper (1987, pp. 255-7, 260-1, 265).
} 
serie causali della materia secondo l'ordine dovuto, in modo tale che la loro concatenazione adeguata risulti in un nuovo individuo della stessa specie. Le serie causali non potrebbero concatenarsi nell'ordine dovuto solo in conseguenza ai loro moti necessari, dal momento che esse si combinano secondo un ordine accidentale ed estrinseco ad essi. In questo consiste l'irriducibilità delle funzioni proprie dell'individuo alla pura materia ${ }^{28}$ e l'inseparabilità delle attività spirituali dal corpo naturale.

La materia, per lo meno in uno dei suoi significati ${ }^{29}$ è condizione imprescindibile alla perfezione ultima della forma e si trova immanentemente integrata come elemento costituente. In verità, la relazione fra materia e forma comporta una stratificazione che si articola in varie tappe ${ }^{30}$, le quali includono, tra il corpo come organismo e i quattro elementi, le parti organiche e le parti omeomere. La materia nell'ultima tappa di questa stratificazione, ossia la materia propria (oikeia hule) ${ }^{31}$, designa un congiunto di forme che, immanenti nella forma specifica dell'uomo, si presentano come condizioni necessarie (sunaition) alla costituzione di

\footnotetext{
${ }^{28}$ Vd. Angioni (1999); Balme (1987, pp. 281-5); Cooper (1987), Charles (1991, pp. 121, 125-6). Un approccio eccellente nella "compatibilità" tra necessità assoluta della materia e necessità ipotetica è offerto da Charles (1988), Lewis (1988), Matthen (1989). Alcuni, come Sorabji (1980, pp. 158-159) e Irwin (1988, p. 102) tendono a concepire la compatibilità in termini puramente epistemologico e/o pragmatici. Altri, come Gotthelf (1987), sono riluttanti ad ammetterla e insistono a discernere nelle forme teleologiche della natura vivente un "potenziale irruducibile". Per una sintesi di questo dibattito, vd. Bradie\&Miller (1984).

${ }^{29}$ Sul duplice senso del termine $0 \ddot{\lambda} \eta$ vd. Angioni (2007, pp. 210-250). Angioni distingue con ammirevole chiarezza un senso di $0 \lambda \eta \eta$ con il quale Aristotele designa il concetto proprio di materia e un senso di $\tilde{u} \lambda \eta$ come materia correlata ad una certa forma. In questo secondo senso, decisivo per la nostra argomentazione, "materia" designa certe forme che, in vista di determinate correlazioni, assumono la funzione di materia, ossia di elemento capace di ricevere la forma superiore di cui sono materia.

${ }^{30}$ Vd. Furth (1988, pp. 76-83).

${ }^{31}$ Aristotele, (Metaph. VIII 4, 1044a 15-20, in particolare, a18).
} 
quest'ultima. Le argomentazioni decisive a questo proposito si trovano nella conclusione del libro VII della Metafisica, in cui il filosofo afferma che la materia, in unione con la forma, è elemento intrinseco dell'ousia sensibile.

Si potrebbe, a questo punto, comodamente concludere che, dal momento che il dualismo mente-corpo in Aristotele non esiste, non ha senso porsi il problema se esista una filosofia della mente. Nell'ultimo capitolo della sua opera Physicalism, K. V. Wilkes scrive: "Aristotle and all Greek Philosophers before him lacked of the concept of a "mind"; lacking any such notion, they lacked too the concept of "the mental"; and hence they had not mind-body problem. Within Aristotle's psychology the relation of the mental to the physical cannot even be posed" (WILKES, 1978, p. 155). A Wilkes fa eco Ackrill: "The idea of a pure intellect literally separable from the body is difficult to understand, and difficult to reconcile with the idea of Aristotle's philosophy" (ACKRILL, 1981, p. 62). Questa idea era stata sviluppata da D. W. Hamlyn nella sua introduzione al De Anima (HAMLYN, 1968, Introduzione). A noi pare, però, che tale soluzione assunta in modo radicale abbia il fascino del paradosso.

Infatti, mentre Aristotele sembra riporre molta fiducia nell'unità delle creature viventi, nondimeno insiste sulla differenza fra $\mathrm{i}$ due aspetti del sinolo in particolare negli scritti biologici. In De Partibus Animalium il filosofo afferma che l'anima è ciò per cui un corpo esiste (ARISTOTELE, PA I 5, 645 19 ); in diversi passi del libro primo dello stesso trattato lo Stagirita dichiara che compito dello studioso della natura è indagare l'anima e il composto piuttosto che il corpo e la materia (ARISTOTELE, PA I 1, 641²9; cfr. I 5, 64530); nell'opera Sul Sogno e sulla veglia Aristotele dichiara 
che la percezione è attività non solo dell'anima ma anche del corpo (ARISTOTELE, Sul Sogno e sulla veglia 454a); infine, ben consapevole dei pericoli dell'ilozoismo, il pensatore greco critica alcuni dei suoi predecessori per il fatto che non pongono la dovuta attenzione alla differenza fra gli enti dotati di anima e quelli che ne sono privi (DA II 1, 411 7 ; a14; cfr. HA I 1, 588 4 ; PA IV 5, 681 12 ).

È innegabile che una delle caratteristiche più originali della teoria aristotelica sia stata quella di avere introdotto l'idea dell'unione e correlazione fra anima e corpo. Tuttavia sembra che, per vincere questa battaglia contro Platone e contro gli atomisti, il filosofo abbia dovuto distinguere con precisione le due componenti prima di insistere sulla loro unità, la quale, infine, ha prevalso.

Inoltre, si potrebbe con una insistenza e una convinzione pari a quelle manifestate da Wilkes e Ackrill sostenere che il termine greco nous esprime precisamente il nostro concetto di "mente". Del resto, Aristotele parla del nous come di una parte dell'anima e sottolinea il fatto che esso è differente dalle altre parti dell'anima: "Per ciò che concerne l'intelletto e la capacità teoretica, nulla ancora è evidente, ma sembra trattarsi di un altro tipo di anima, e solo questa ammette la possibilità di essere separata, proprio come ciò che è eterno è se-

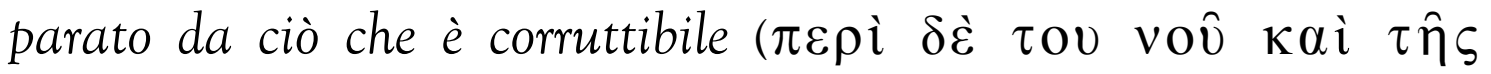

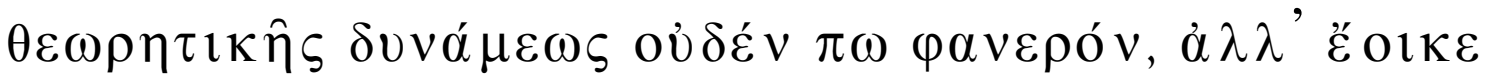

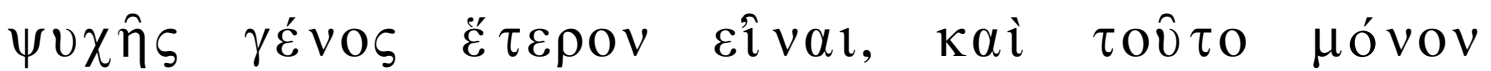

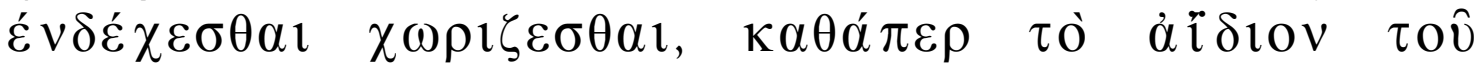
$\varphi \theta \alpha \rho \tau$ ov̂." (DA II 2, 413 $23-25)$. Aristotele non si pronuncia in modo decisivo in relazione alla parte intellettiva dell'anima, che sembra creargli un certo imbarazzo. Per lo meno a prima vista, l'anima intellettiva sembra sottrarsi al progetto hylemorfico che il filosofo greco estende a tutta la 
propria filosofia della natura ${ }^{32}$.

Se non è questo il problema del rapporto mente-corpo, di cosa si tratta allora?

\section{IL PROBLEMA DEL NOUS}

Le osservazioni che Aristotele fa a proposito della facoltà noetica fanno risorgere la questione se il filosofo greco, in fin dei conti, non si sia sentito forzato a reintrodurre un soggetto separato, nella forma di un intelletto, per rendere conto del pensiero, o almeno di un certo tipo di pensiero (DA I 4,408 18 -29). Noi non siamo certo nella felice posizione di risolvere il problema, ma in fondo accarezziamo l'idea che Aristotele non fosse affatto incline a rifiutare la soluzione hylemorfica per rendere ragione di questa particolare facoltà umana.

Anzitutto, non c'è alcun dubbio sul fatto che l'umano intelletto presupponga l'attività di un intelletto immateriale che non dipende in alcun modo dal corpo ${ }^{33}$. Questo è il noto intelletto attivo di De Anima III 5 (DA III 5, 430 26 29; III 5, 430 14-18; cfr. II 5, 417 ${ }^{\mathrm{b}} 23-24$; III 7, 431 ${ }^{\mathrm{b}} 16-17$ ). La sua attività chiaramente non è esercizio di una facoltà naturale e perciò non può essere spiegata allo stesso modo. Ma ciò non comporta che la dottrina dell'intelletto attivo sia incompatibile con la teoria hylemorfica del rapporto anima/corpo. Potremmo assumere, ponendoci in questo

\footnotetext{
${ }^{32}$ Si tratta di un problema classico formulato da Nuyens (1973) e recentemente ripreso da Zingano (1998).

${ }^{33}$ Aristotele, (DA II 2, 413"24-25): "Per quanto riguarda l'intelletto e la capacità di pensare, nulla è evidente, ma sembra trattarsi di un altro tipo di anima, e solo questo ammette la separazione, così come ciò che è eterno è separato da ciò che è corruttibile"; (I 4, 408 25-28): "In relazione all'intelletto, forse si tratta di qualche cosa di più divino ed impassibile".
} 
modo al seguito di una lunga tradizione di interpreti, che questo intelletto attivo non sia l'intelletto umano, quindi non ci sia ragione di intenderlo allo stesso modo.

Ma anche mettendo da parte l'attività dell'intelletto attivo resta pur sempre da giustificare l'intelletto passivo. Fin dal principio del De Anima Aristotele solleva la questione se l'attività del pensare (noein) sia da considerare alla stregua delle altre facoltà dell'anima (DA III 4, 429a13-23; III 4, $\left.429^{b} 35-430^{a} 2\right)$. E in questo caso egli fa chiaramente riferimento all'intelletto umano. In questi passi Aristotele sembra essere oscillante, e quel che è peggio pare incline a ritenere che l'attività del noein non coinvolga il corpo allo stesso modo in cui esso è coinvolto nell'atto delle altre affezioni dell'anima. Perciò si è inclini facilmente a credere che neppure l'intelletto passivo possa essere spiegato secondo le linee tracciate precedentemente. Ma giungere troppo precipitosamente a questa conclusione sarebbe, forse, un errore.

Anzitutto si tratta di precisare, per quanto sia possibile, cosa sia il nous.

A questo proposito la risposta più immediata non viene dal De Anima bensì dagli Analitici. In seguito ad una accurata lettura di Analitici Posteriori II 19, e dopo la critica di Barnes al modello dell'intelletto inteso come "intuizione mentale" (1975), il nous viene tendenzialmente inteso come la disposizione propria a conoscere certi principi (ARISTOTELE, A Po $100^{\mathrm{b}} 1-2$ ), certe proposizioni prime e indimostrabili. Aristotele talvolta menziona nei capitoli centrali degli Analitici Secondi che dei principi non si dà conoscenza dimostrativa (ARISTOTELE, A Po 71 ${ }^{\mathrm{b}} 16$; $72^{\mathrm{b}} 18$ $\left.25 ; 88^{\mathrm{b}} 35\right)$, senza pronunciarsi chiaramente su di un tipo di conoscenza alternativo. Il filosofo lascia al capitolo finale della sua opera una duplice incombenza: sapere come que- 
sti principi si rendono noti e quali siano le disposizioni (hexeis) che ci rendono capaci di conoscere i principi (A Po II $\left.19,99^{\mathrm{b}} 15-19\right)$, in particolare se esse sorgono in noi non essendo immanenti alla natura umana o, al contrario, se siano immanenti ma rimangono per lungo tempo inconsce. La soluzione di Aristotele sarà di non scartare nessuna delle due ipotesi, ma di approfittare di elementi dell'una e dell'altra utili a risolvere il problema. Egli pretende mostrare che il coglimento dei principi si radica nella sensazione, ossia mostra come possiamo conoscere principi sconosciuti per mezzo di una conoscenza antecedente nata dalle sensazioni. Del resto, la capacità di conoscere i principi richiede una certa dunamis che, il filosofo precisa, si trova per natura in tutti gli animali: la sensazione (aisthesis) ${ }^{34}$. A partire dalla sensazione Aristotele spiega la genesi delle altre disposizioni, ossia il coglimento dei principi.

Tutti gli animali sono dotati di sensazione, ma solo alcuni sono in grado di mantenere un segno (mone) comune di ciò che hanno percepito più volte; fra questi ultimi animali, solo alcuni sono capaci di notare una differenza tra questi diversi segni comuni, rendendo così possibile il sorgere della razionalità (logos). Illustrando la genesi della conoscenza, lo Stagirita dirà che dalle sensazioni simili si produce una esperienza (empeiria). L'esperienza riunisce in una unica rappresentazione universale una molteplicità di sensazioni e ricordi simili. Tra questi universali, alcuni già rappresentano reali principi scientifici. Per questo Aristotele conclude che, a partire dall'esperienza, sorge "principio di

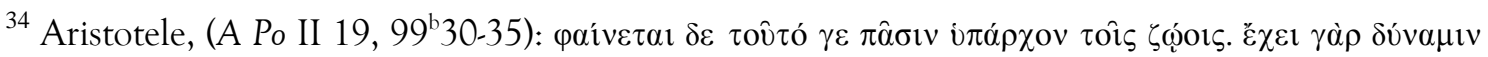

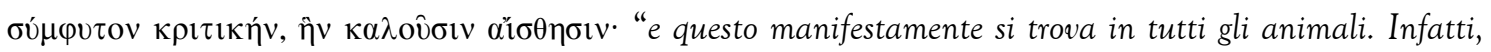
tutti hanno una capacità discriminativa innata, che si chiama sensazione”.
} 
arte e scienza, dell'arte se riguarda il venire all'essere, ma della

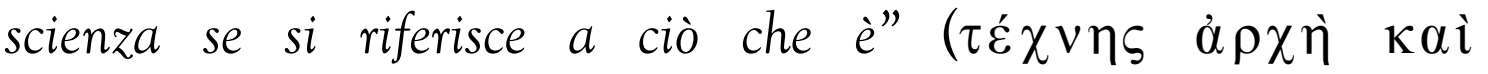

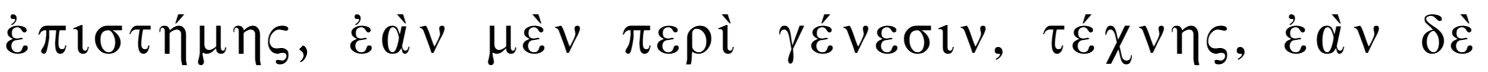

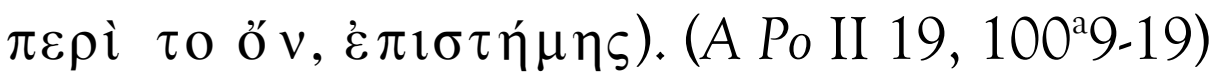

Perciò, nella misura in cui abbiamo una facoltà immanente capace di generare una certa disposizione, la disposizione è immanente in noi; ma, dal momento che la sensazione non è identica alla disposizione ed è più oscura di essa, la disposizione non è immanente. Il paragrafo successivo (A Po, II 19, 100 $14-\mathrm{b} 5$ ), forse il più difficile dell'intero capitolo, descrive il processo di astrazione e generalizzazione grazie al quale si passa da una molteplicità di sensazioni particolari ad una nozione universale. È sufficiente che da un complesso confuso di sensazioni si ricavi un indifferenziato (adiaphoron), nel quale sia possibile distinguere alcune caratteristiche comuni, mediante le quali esso venga distinto dal complesso indifferenziato delle altre sensazioni (Cfr. BOLTON, 1993). La sensazione a partire dalla quale arriviamo all'universale porta con sé, benché in una forma ancora indistinta, l'universale. Ora, Aristotele sembra identificare l'intero processo di generalizzazione e astrazione descritto con l'induzione (epagoge): "stando cosi le cose, è chiaro che è necessario conoscere $i$ principi per induzione. Infatti è così che la sensazione produce in noi l'universale"

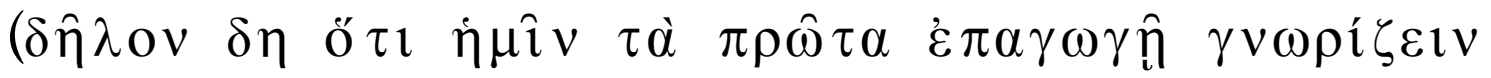

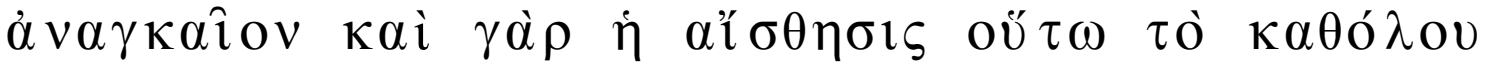
$\dot{\varepsilon} \mu \pi$ oı $\varepsilon \hat{\imath}$.) (ARISTOTELE, A Po II 19, 100³-5)

L'ultimo paragrafo, infine, è consacrato alla disposizione che ci permette di conoscere i principi. Aristotele la presenta in modo molto conciso, quasi solo nominandola, ma senza chiarire il suo funzionamento e la relazione che mantiene con il processo di induzione appena descritto. Se- 
guiamo l'argomentazione di Aristotele: tra le disposizioni (exeon) dianoetiche che sono sempre vere, ci sono solo la scienza (episteme) e l'intelligenza (nous); i principi sono più esatti che le dimostrazioni e non sono oggetto di scienza, ma di una disposizione dianoetica più esatta della scienza; l'intelligenza è più esatta della scienza, perciò ad essa compete la conoscenza dei principi. L'intelligenza, pertanto, è la disposizione che ci rende noti i principi della scienza, essendo essa principio della scienza (A Po II 19, 100 5-17).

Aristotele chiude così la sua opera che, se non chiarisce il modo di operare del nous, lascia indubitabile il fatto che l'intellezione dei principi non solo richiede la sensazione, ma si fondamenta precisamente in questa conoscenza sensibile anteriore e presente in tutti gli animali.

\section{CONCLUSIONE}

Possiamo riconoscere, al termine di questa analisi, che Aristotele condivide con i moderni la convinzione che gli oggetti naturali e il loro comportamento proprio non possono essere spiegati nei termini dei materiali costituenti e delle proprietà di essi, ma egli ritiene, d'altra parte, che una spiegazione in termini "naturali" sia sufficiente e non richieda l'intervento di altri elementi astratti dotati di facoltà particolari. Ė importante non sottostimare che la differenza fondamentale fra Aristotele e i moderni non risiede tanto nei dettagli di una teoria dei materiali costituenti degli enti e delle loro proprietà, bensì nella risposta alla questione su quale teoria provveda a fornire una esauriente comprensione degli enti naturali e del loro comportamento. Né ciò che distingue la posizione di Aristotele da quella moderna è un disaccordo sullo statuto dei fenomeni mentali, bensì un di- 
saccordo su cosa sia un ente naturale e come possa essere spiegato il suo comportamento. Causa dell'essere e delle manifestazioni dell'essere vivente è, per Aristotele, la "natura" di un corpo animato, ossia la sua ousia. Per gli enti sensibili, causa dell'essere e ousia dell'ente è l'anima, che è una forma capace di sussumere la materia come elemento costituente la sua propria attualità. La "natura" propria di un ente sensibile è in grado di rendere conto di tutte le manifestazioni dell'essere vivente, che Aristotele rifiuta di distinguere in mentali e fisiche. Anche quando lo Stagirita attinge all'apice della conoscenza umana, ossia il coglimento dei principi, egli non fa appello ad una misteriosa intuizione atta a cogliere i principi, bensì ad una disposizione che mette nella condizione di trarre inferenze causali a partire dall'osservazione di certi fenomeni. Questa disposizione non è innata (A Po 99 26-27), bensì si acquisisce affinando le predisposizioni naturali, ossia la sensazione, la percezione, la memoria e l'esperienza in generale (A Po 1003-9).

Perciò è vero che l'esercizio dell'intelletto non è simile all'esercizio delle altre affezioni dell'anima (DA III 4, $429^{\mathrm{a}} 18$ e 24-25), in quanto non comporta il diretto coinvolgimento di un organo corporeo (DA III 4, 429a29-b5; cfr. 429a24-27). Ciononostante, non è possibile esercitare l'attività pensante ammenoché non si sia enti dotati di sensazione e percezione e ammenoché non si sia in grado di ricordare ciò che è stato percepito. Perciò la nostra capacità di pensare presuppone un corpo.

Del resto, l'attività pensante richiede l'uso di immagini di un certo genere (DA I 1, 403 8-9). La memoria, e persino la capacità di pensare gli enti intelligibili (ta noetà) richiedono l'uso di phantasmata e il phantasma è pathos (DA III 3, 
427 $14-16$; III 3, 428 1 -4; III 3, 428 $19-24$; III 3, 428 2 -4; III 3, 429 1-4), ossia è affezione della koinê aisthesis (DA III 8, $\left.432^{\mathrm{a}} 7-9\right)$. Sembra proprio che, in quanto animali senzienti non possiamo pensare neppure gli enti intelligibili, se non attraverso i phantasmata, ossia la base hylemorfica del nostro pensiero (DA III $1,425^{\mathrm{a}} 30^{\mathrm{b}} 1$; III $7,431^{\mathrm{b}} 2$; cfr. III $9,432^{\mathrm{b}} 16$ -

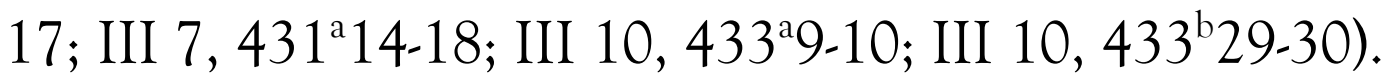

Complessi problemi che il nous comporta e la relazione fra il nous e le altre affezioni dell'anima derivano anche dal tentativo di Aristotele di rendere giustizia della nostra peculiare natura di esseri umani, i quali da una parte condividono il dominio animale; dall'altra in qualche modo lo trascendono e accedono all'ambito noetico che, in termini moderni, si potrebbe indicare come l'ambito della cultura, della scienza e dell'etica, nonché della tecnologia. Non è in quanto animali senzienti che abbiamo accesso a questo dominio, bensì in quanto enti dotati di linguaggio e intelletto. Perciò sembra che la dottrina della psiche umana richieda da un lato una definizione hylemorfica dell'uomo, dall'altra la presenza di un nous incorporeo che trascenda il sinolo di anima e corpo. Ma questo dualismo è solo apparente.

Forse questa idea di incorporeità del nous, della sua separabilità, rappresenta il sostituto del mito platonico del Fedro. Entrambe le dottrine possono essere interpretate come metafore utili a rappresentare quel qualcosa in più che solo l'intelletto umano ha e che non è forse ancora stato interamente capito. Crediamo che nessuna scoperta nel campo della neurofisiologia possa fornire un resoconto adeguato del fenomeno culturale, qualora questo termine racchiuda significati linguistici e credenze proposizionali. L'insieme della cultura comprende lo sviluppo preistorico 
della tecnologia, le forme dell'arte simbolica, le istituzioni sociali, ossia tutto ciò che rappresenta il risultato dell'utilizzo del principio noetico. Nessun modello neurofisiologico, per quanto adeguato, crediamo riesca a rendere conto precisamente di questi fenomeni propriamente intellettuali.

E forse è inevitabile che sia così, perché è grazie all'intelletto che ogni altra cosa viene compresa e spiegata.

Abstract: In the three books of the De Anima Aristotle ranges over a diverse array of philosophical and scientific topics, such as the nature of life, selfmovement, the senses, perception, imagination, thought, and the relation between mind and body. As a result, this work may seem to be a strange collection of only marginally related philosophical and biological topics given our modern sensibilities. Nonetheless it is united by Aristotle's basic concern for the nature and functioning of life in all its diverse forms. This inquiry offers modern readers significant insight into contemporary debates in the philosophy of mind.

Key-words: soul, mind, body, thought.

\section{REFERÊNCIAS}

\section{OPERE DI ARISTOTELE}

Angioni, L., Aristóteles, As Partes dos Animais, livro I. Cadernos de Historia e Filosofia da Ciência, série 3, v. 9, n. especial, 1999.

Apostle, H., Aristotle's On The Soul. Grinnell, Iowa: The Peripatetic Press, 1981.

Balme, D.M., Aristotle, De Partibus Animalium I and De Generatone Animalium (with passages from II 1-3), with a Report on recent Work and an Additional Bibliography by A. Gotthelf, Oxford: Clarendon Press, 1992. 
Barnes, J., Aristotle's Posterior Analytics, Oxford: Clarendon Press, 1975.

Lawson-Tancred, H., Aristotle, De Anima (On the Soul), translated with introduction and notes, New York: Penguin, 1986.

\section{STUDI}

Ackrill, J. L., Aristotle's Definition of psuche, in Barnes, Schofield, Sorabji (ed.), Articles on Aristotle, vol. 4, London: Duckworth, 1979, pp. 65-75.

Angioni, L., A noção aristotélica de matéria, "Cadernos de História e Filosofia da Ciência”, III, 17, 2007.

Balme, D.M. Teleology and necessity, in Gotthelf, A. and Lennox, J. (edd.), Philosophical Issues in Aristotle's Biology, Cambridge: Cambridge University Press, 1987, pp. 275285.

Barnes J., Schofield J., Sorabji R., (eds.), Articles on Aristotle: Psycology and Aestetics, (vol. III), London: Duckworth, 1979.

Bernard, W., Hylomorfism, "Oxford Studies in Ancient Philosophy", IV, 1986, pp. 189-99.

Berti, E., Aristotele e il "Mind-Body Problem", "Iride", 1988, pp. 43-62.

Bolton, R., Aristotle's definition of the soul: De Anima II, 1-3, "Phronesis", XXXIII, 1978, pp. 258-278.

Bolton, R., Division, Définition et Essence dans la science aristotélicienne, "Revue Philosophique de la France et de 
l'étranger", Avril-Juin n. 2, PUF, 1993, pp. 197-222.

Bos, A.P., The soul and its Instrumental Body. A reinterpretation of Aristotle's Philosophy of Living Nature, LeidenBoston: E. J. Brill, 2003.

Botter, B., La necessità naturale in Aristotele, Napoli: Loffredo Editore, 2009.

Bradie, M., \& Miller, F.D., Teleology and Natural Necessity in Aristotle, "History of Philosophy Quarterly", vol.1, n. 2, 1984, pp. 133-146.

Brunschwig J., La forme, prédicat de la matière?, in Aubenque, P. (ed.), Etudes sur la Métaphysique d'Aristote, Actes du VI Symposium Aristotelicum, Paris: Vrin 1979, pp. 131-158.

Burnyeat, M.F., Is an Aristotelian Philosophy of Mind still Credible?", in M.C. Nussbaum, A.O. Rorty (edd.), 1992, pp. 15-26.

Charlton, W., Aristotle's Definition of Soul. In Aristotle's De Anima in Focus. Edited by Michael Durant. New York: Routledge, 1993.

Charles, D., Teleological Causation in the Physics, in Judson, L. (ed.), Aristotle's Physics, Oxford: Clarendon Press, 1991, pp. 101-128.

Charles, D., Aristotle on Hypothetical Necessity and Irreducibility, "Pacific Philosophical Quarterly", vol. 69, n. 1, 1988, pp. 1-53.

Cohen, S.M., Hylomorfism and Functionalism, in M.C. Nussbaum, A.C. Rorty (edd.), 1992, pp. 57-73. 
Cooper, J.M., Hypothetical necessity and natural teleology, in Gotthelf, A. and Lennox, J. (edd.), Philosophical Issues in Aristotle's Biology, Cambridge: Cambridge University Press, 1987, pp. 243-274.

Descartes, R., Descartes: Selected Philosophical Writings. Translated by John Cottingham, Robert Stoothoff, and Dugald Murdoch. New York: Cambridge UP, 1988.

Furth, M., Substance, Form and Psyché: an Aristotelian Metaphysics, Cambridge: Cambridge University Press, 1988.

Gotthelf, A. (ed.), Aristotle on Nature and Living Things, Pittsburg-Bristol: Mathesis Publications, 1985.

Gotthelf, A., Lennox, J.G, (edd.), Philosophical Issues in Aristotle's Biology, Cambridge: Cambridge University Press, 1987.

Gotthelf, A., Aristotle's Conception of final causality, in Gotthelf, A. \& Lennox, J. (edd.), Philosophical Issues in Aristotle's Biology, Cambridge: Cambridge University Press, 1987, pp. 167-198.

Green, C., The Thoroughly Modern Aristotle: Was he really a functionalist? Presented at Aristotle and Contemporary Science, Thessaloniki, Greece in September 1997.

Hammond, W., Aristotle's Psychology. New York: MacMillian,1902.

Hardie, W.F.R., Aristotle's Treatement of the relation between Soul and the Body, "Philosophical Quarterly" 14, 1984, pp. 53-72. 
Hartmann, E., Substance, Body and Soul, Princeton: Princeton University Press, 1978.

Irwin, T., Aristotle's First Principles, Oxford: Clarendon Press, 1988.

Jaulin, A., L'Âme et la vie selon Aristote, "Kairos", IX, 1997, pp. 121-40.

Kim, J., Philosophy of Mind. Boulder: Westview Press, 1998.

Kuhn, J., Can Substance Be Predicated of Matter?, "Archiv für Geschichte der Philosophie" 60, 1978, pp. 140-159.

Lennox, J.G., Aristotle's Philosophy of Biology. Studies in the Origin of the Life Science, Cambridge: Cambridge University Press, 2001.

Lewis, F., Teleology and Material/Efficient Causes in Aristotle, "Pacific Philosophical Quarterly", vol. 69, n. 1, 1988, pp. 54-98.

Lewis, F., Substance and Predication in Aristotle, Cambridge: Cambridge University Press 1991.

Lloyd, G.E.R., Owen, G.E.L. (eds.), Aristotle on Mind and the Senses: Proceedings of the Seventh Symposium Aristotelicum, Cambridge: Cambridge University Press, 1978.

Loux, M., Primary Ousia: an Essay on Aristotle's Metaphysics $Z$ and H, Ithaca: Cornell University Press 1991.

Matthen, M., The four Causes in Aristotle's Embriology, in Penner, T. Eु Kraut, R. (edd.), Nature, Knowledge and Virtues, "Apeiron" 22, n. 4 (numero speciale), 1989, pp. 159. 
179.

Menn, S., Aristotle's Definition of Soul and the Programme of the De Anima, "Oxford Studies in Ancient Philosophy", XXII, 2002, pp. 83-139.

Miller Jr., F.D., Aristotle's Philosophy of Soul, "The Review of Metaphysics", LIII, 1999, pp. 309-37.

Nussbaum, M.C., Rorty, O.A., (edd.), Essays on Aristotle's De Anima, Oxford: Oxford Clarendon Press, 1992.

Nussbaum, M. C., Aristotelian Dualism : a Reply to Robinson, "Oxford Studies in Ancient Philosophy", II, 1984, pp. 198-207.

Nussbaum, M. C., Aristotle on teleological explanation in Aristotle's MA, Princeton: Princeton University Press, 1978, pp. 59-106.

Nussbaum, M. C., Substance, Body and Soul: Aristotelian Investigations, "Journal of Philosophy", vol. 77, n. 6, 1980, pp. 355-365.

Nuyens, F., L'evolution de al psychologie chez Aristote, Louvain-La-Neuve: Institut Supérior de Philosophie, 1973.

Sauvé, S., Aristotle, Teleology and Reduction, "Philosophical Review" 101, n. 4, 1992, pp. 791-825.

Searle, J., The Rediscovery of the Mind. Cambridge: The MIT Press, 1992.

Shields, Ch., Soul and Body in Aristotle, "Oxford Studies in Ancient Philosophy” VI, 1988, pp. 103-137. 
Sorabji, R., Necessity, Cause and Blame, London: Duckworth 1980.

, Body and Soul in Aristotle. In Aristotle's De Anima in Focus. Edited by Michael Durant. New York: Routledge, 1993.

Waterloo, S., Nature, Change and Agency in Aristotle's Physics, Oxford: Clarendon Press 1982.

Wilkes, K. V., Physicalism, New York: Atlantic Highlands, 1978.

Wittgenstein, Remarks on the Foundation of Mathematics, transl. G.E.M. Anscombe, Cambridge, 1956.

Whiting, J., Living Bodies, in M.C. Nussbaum, A.O Rorty (edd.), 1992, pp. 75-92.

Zingano, M., Razão e Sensação em Aristóteles: um Ensaio sobre De Anima III 4-5, Porto Alegre: L\&PM editores, 1998. 